\title{
Elastic Properties of Supported Polycrystalline Thin Films and Multilayers : an X-ray Diffraction Study
}

\author{
P. Goudeau ${ }^{1}$, P. Villain ${ }^{1}$, N. Tamura ${ }^{2}$, P.-O. Renault ${ }^{1}$, K.F. Badawi ${ }^{1}$ and H. \\ A. Padmore ${ }^{2}$ \\ ${ }^{1}$ Laboratoire de Métallurgie Physique - UMR 6630 CNRS - Université de Poitiers, SP2MI, Bvd \\ Marie et Pierre Curie, B.P. 30179, 86962 Futuroscope Chasseneuil Cedex, France \\ ${ }^{2}$ Advanced Light Source, Lawrence Berkeley National Laboratory, 1 Cyclotron Road, Berkeley, CA \\ 94720, USA
}

Keywords: X-ray diffraction, residual stresses, elastic constants, microstructure, thin films, multilayers

\begin{abstract}
Numerous experimental and theoretical studies have shown that thin film elastic behavior may be different from the bulk one due to size effects related to grain boundaries, free surfaces and interfaces. In addition, thin films often present high compressive residual stresses which may be responsible of thin film buckling. These two features will be discussed in this communication through recent xray diffraction experiments: in situ tensile testing for elastic constant analysis and scanning x-ray micro diffraction for stress relaxation measurements associated with film buckling.
\end{abstract}

\section{Introduction}

Thin films deposited on non epitaxial substrates by ion beam sputtering often exhibit large compressive residual stresses and nanocrystalline structures. Theses features are tightly controlled by the deposition process (mainly the deposited atom energy) and confer to the films interesting physical properties [1]. In order to get a better understanding of the possible strain effect on physical properties, the determination of the intrinsic mechanical properties of the film in relation with the microstructure is then necessary. Among the most widely used methods to study thin film mechanical properties, $x$-ray diffraction (and in particular the $\sin ^{2} \psi$ method) is the unique non destructive and phase selective technique which allows to study both the micro structural and mechanical state of the diffracting phase [2]; the inter planar atomic distance is used as an internal strain gauge.

However, $x$-ray diffraction (XRD) is difficult to use in low dimensional systems because the diffracted intensities are weak due to the reduced thicknesses and nanocrystalline character of such materials. These problems may be solved using intense x-ray sources such as synchrotron radiation (S.R.). In addition to the high flux characteristic of S.R. facilities, the wide wavelength spectra and the optics which are now available on $3^{\text {rd }}$ generation S.R. beam lines only allow to perform specific XRD experiments which are not possible with classical x-ray sources in laboratories. This paper deals with the two following x-ray diffraction experiments which have been developed for studying thin film mechanical properties:

In situ tensile testing for elastic constant determination: due to the particular microstructure (defects, grain size effect), the thin film elastic constants may differ from the bulk material ones (if there exist!). We applied in situ strains to $150 \mathrm{~nm}$ W thin films and W/Cu multilayers with low period $(\Lambda=3 \mathrm{~nm})$ in order to extract $\mathrm{W}$ elastic constants from X-ray diffraction data,

$X$-ray micro beam for residual stress mapping: depending on the deposited thickness and substrate nature, relaxation of the stored elastic energy in the films may lead to thin film buckling. $\mu$-XRD local stress measurements with micrometer spot sizes have been done on two dimensional (2D) gold blisters and one dimensional (1D) tungsten wrinkles. 


\section{Measuring thin film and multilayer elastic constants by coupling in situ tensile testing with X-ray diffraction}

Theoretical studies concerning nanocrystalline materials [3,4] predict noticeable modifications of their elastic properties compared to the bulk ones due to a high volume proportion of surface and interface atoms, as well as a high point defect density. Grain boundaries, free surfaces and interfaces induce important alterations of cohesion energies. Numerous experimental studies have evidenced a very probable softening of elastic constants in nanostructured materials, in particular the $\mathrm{C}_{44}$ stiffness coefficient in multilayers with very low $(\Lambda \leq 3 \mathrm{~nm})$ modulation wavelength [5-7]. In previous papers, we described an experimental technique combining in situ tensile testing and x-ray diffraction to extract the Poisson's ratio [8] and the Young's modulus of supported thin metallic films [9] and multilayers [10]. This diffractometric method differs from all the other mechanical, vibration, and acoustic techniques since it allows to measure average in-grain strains, and thus intragranular elastic constants.

The technique used here, based on the well known " $\sin ^{2} \psi$ method" [2], consists of applying a uniaxial tensile force to the film/substrate system, in situ in an x-ray diffractometer (Fig. 1).

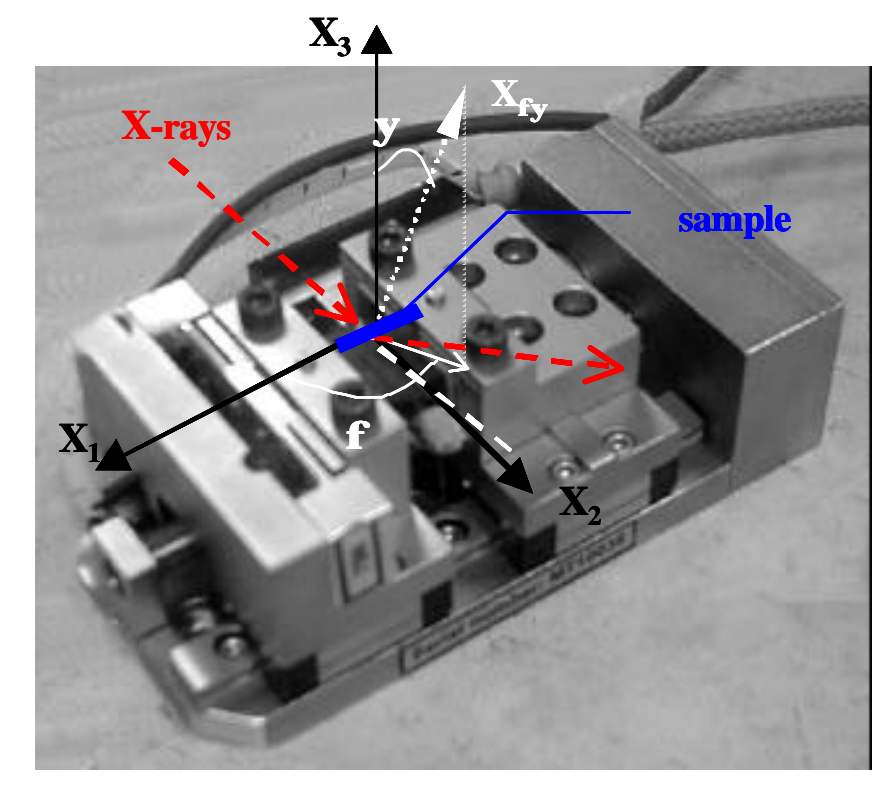

Figure 1. Deben ${ }^{\mathrm{TM}}$ tensile tester with the sample coordinate $\left(\mathrm{X}_{1}, \mathrm{X}_{2}, \mathrm{X}_{3}\right)$ and the $\mathrm{x}$-ray measurement direction $\mathrm{X}_{\phi \psi}$.

The formalism is very simple in the case of a polycrystalline elastically isotropic material such as tungsten: a linear relation is expected between the shift of each $\{\mathrm{hkl}\}$ peak position and $\sin ^{2} \psi, \psi$ being the angle between the normal to the diffracting planes and the normal to the sample surface. For each applied load value, the $\sin ^{2} \psi$ curves are determined for the tungsten layers. The slope $\mathrm{P}_{1}$, on the one hand, and the intercept $\mathrm{m}_{1}$, on the other hand, of the linear regressions on these experimental data is plotted as a function of the applied load F. Assuming an uniaxial applied stress state (similar Poisson's ratios for the film and the substrate), the mean Young's modulus $\mathrm{E}_{\mathrm{N}}$ of tungsten sublayers can be then calculated by means of the following relation:

$$
\mathrm{E}_{\mathrm{W}}=\frac{1}{\mathrm{t}_{\mathrm{W}}}\left(\frac{1}{\mathrm{~b}\left(\mathrm{P}^{*}+\mathrm{m}^{*}\right)}-\mathrm{E}_{\mathrm{s}} \mathrm{t}_{\mathrm{s}}-\mathrm{E}_{\mathrm{Cu}} \mathrm{t}_{\mathrm{Cu}}\right)
$$


where $b$ is the sample width, $t_{\mathrm{s}}$ the substrate thickness, $t_{\mathrm{W}}$ (resp. $\mathrm{t}_{\mathrm{Cu}}$ ) the total thickness of the $\mathrm{W}$ (resp. $\mathrm{Cu}$ ) sublayers, $\mathrm{E}_{\mathrm{s}}\left(\mathrm{resp} . \mathrm{E}_{\mathrm{Cu}}\right.$ ) the substrate (resp. copper) Young's modulus, $\mathrm{P}^{*}$ (resp. $\mathrm{m}^{*}$ ) the slope of the $\left(\mathrm{P}_{1}, \mathrm{~F}\right)$ linear curve (resp. the $\left(\mathrm{m}_{1}, \mathrm{~F}\right)$ curve).

The mean Poisson's ratio $v_{\mathrm{W}}$ of tungsten sublayers is simply deduced from a combination of $\mathrm{P}^{*}$ and $\mathrm{m}^{*}$ :

$$
v_{\mathrm{W}}=-\frac{\mathrm{m}^{*}}{\mathrm{P}^{*}+\mathrm{m}^{*}} \text {. }
$$

It is worth noting that the determination of $v_{\mathrm{W}}$ does not need any numerical value concerning geometrical nor mechanical properties of the various parts of the system. For the Young's modulus measurement, the substrate Young's modulus and the copper one must be known, as well as the respective thickness of the three constituents.

W/Cu multilayers were deposited onto a $127.5 \mu \mathrm{m}$ thick polyimide $\left(\operatorname{Kapton}^{\circledR}\right)$ dogbone substrate by ion beam sputtering at room temperature, tungsten being the first deposited layer. The in-plane sample dimensions were $7.4 \times 3 \mathrm{~mm}^{2}$. The total thin film thickness ranged from 220 to $240 \mathrm{~nm}$. Before performing the in situ tensile tests, several characterization experiments have been done for determining the modulation wavelength $\Lambda$ (thickness of one W/Cu bilayer), atomic proportions of tungsten and copper, and then the mean thickness of tungsten $\left(\mathrm{e}_{\mathrm{W}}\right)$ and copper $\left(\mathrm{e}_{\mathrm{Cu}}\right)$ individual sublayers assuming the density of each material is equal to the bulk one. $t$ should be noticed that nanostructured copper and tungsten sublayers may be less dense than the bulk materials as the modulation wavelength $\Lambda$ decreases. This effect implies small variations on the $e_{\mathrm{Cu}}$ and $\mathrm{e}_{\mathrm{W}}$ values when considering the density ratio of both elements in the thickness calculation and thus slightly influences the precision on the modulus measurement (Eq. (1)).

The in situ tensile tests were performed at the French synchrotron radiation facility L.U.R.E. (Orsay, France) on the H10 and DW22 beam lines where we took advantage of the high X-ray beam intensity and quality, as well as the large wavelength range accessible. For each specimen, several increasing strains were applied and $\mathrm{x}$-ray diffraction measurements were realized in each case.

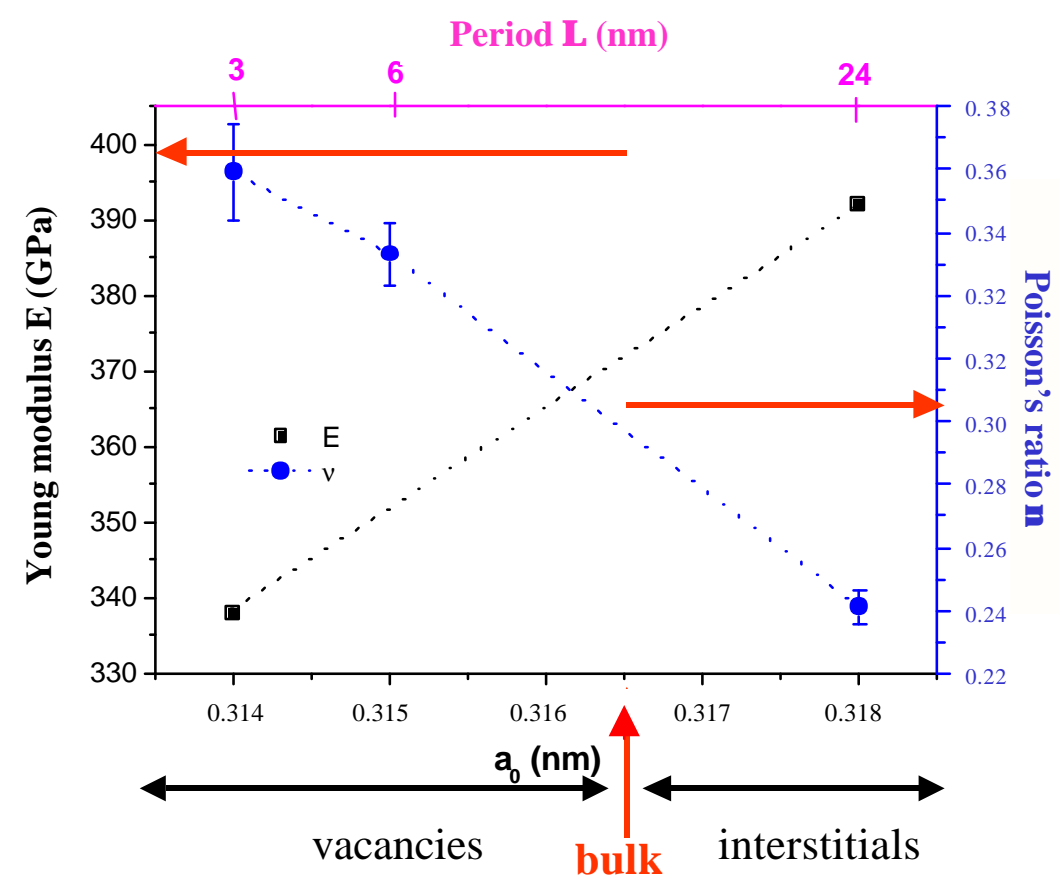

Figure 2. Evolution of the elastic constants of $\mathrm{W}$ sublayers as a function of the period thickness. 
The measured values for tungsten constants are plotted in Fig.2 as a function of the stress free lattice parameter. The results clearly demonstrate that the elastic constants are sensitive to the microstructure: in the " $3.1 \mathrm{~nm}$ " multilayer, the Poisson's ratio $(0.359)$ is larger than the bulk one $(0.284)$ while it is smaller in the " $24.0 \mathrm{~nm}$ " multilayer (0.221); concerning the Young's modulus, a noticeable softening is evidenced in the case of the small period multilayer $(\mathrm{E}=338 \mathrm{GPa})$ while in the other one, tungsten modulus $(\mathrm{E}=392$ $\mathrm{GPa}$ ) is equal to the bulk value (400 GPa) within the uncertainties. It should be kept in mind that the values obtained via $\mathrm{x}$-ray diffraction measurements describe the intragranular elastic behavior of tungsten layers.

\section{Micron-scale xray diffraction measurement of stress relaxation associated with buckling in compressed thin films}

Physical Vapor Deposition (PVD) techniques are widely used in related industries and resulting thin films bonded on non epitaxial substrates generally exhibit large residual stresses due to the mismatch between the film and the substrate. Their structure, morphology - grain size and stress state are tightly controlled by the deposited atom energy. For instance, ion beam sputtering induces nanometric grain sizes, strong compressive residual stresses and large amount of unit cell defects. A compressive in-plane stress can spontaneously lead the film to buckle in order to relieve this stress; this phenomenon generally happen when the film deposited under vacuum is submitted to the atmospheric pressure outside the deposition chamber. Buckling patterns could have a simple shape like circular blisters ( $\mathrm{Au}$ on $\mathrm{Si}$ ) or more complex ones like "Telephone Chords" wrinkles (W on Si). These effects are undesirable and much effort was undertaken to theoretically study these phenomena by way of modeling and simulation [11].

The buckling phenomenon is unambiguously attributed to a relaxation of the elastic energy stored in the film. However, the small dimensions of the buckling have so far prevented to obtain direct strain/stress measurements at the buckling location to support these theoretical results. In this work, we apply a scanning $x$-ray microdiffraction $(\mu \mathrm{SXRD})$ technique capable of resolving stress variations at micron level in nanocrystalline samples.

With the advent of high brilliance 3rd generation synchrotron sources and progress in $x$-ray focusing optics, extremely small and intense x-ray beams are now routinely produced at synchrotron facilities and used to investigate materials with unprecedented resolution under a variety of in-situ conditions. The particularity of the ALS microdiffraction beamline holds in its ability to map strain/stress as well as orientation with submicron spatial resolution, using white light, while it is flexible enough to allow for experiments which require monochromatic microbeams [12]. The ALS 7.3.3. X-ray microdiffraction endstation is able to focus X-rays coming from a bending magnet source down to 0.7 micron using a pair of elliptically bent Kirkpatrick-Baez mirrors. For monochromatic beam applications, a four crystal Si(111) monochromator can be inserted in the path of the beam (energy range: $5.5-14 \mathrm{keV}$ ). This arrangement has the property of leading the monochromatic beam on the same path than the white beam, hence illuminating the sample on the same spot. The sample is on a XYZ scanning stage equipped with heating and cooling capabilities and the diffraction data are collected using an X-ray CCD detector (Bruker SMART 6000, active area of $9 \mathrm{x} 9 \mathrm{~cm}^{2}$ ).

The applications conducted so far at this end-station range from the study of the mechanical properties of composite materials and Microelectromechanical system (MEMS) devices to the characterization of biomaterials, superconducting films, and to mineralogy, geology, environmental and earth sciences [13, 14].

For our purpose, investigated samples were $300 \mathrm{~nm} \mathrm{~W}$ polycrystalline thin films deposited at room temperature by ion beam sputtering technique on a $650 \mu \mathrm{m}$ thick (100) Si wafer covered with native oxide. Details concerning sample preparation and XRD measurements may be found in ref. 15. Fig 3(b) shows the stress map as a result over a $60 \mu \mathrm{m}$ x $140 \mu \mathrm{m}$ area of buckling on a $300 \mathrm{~nm}$ thick $\mathrm{W}$ film on Si. The $\mu \mathrm{SXRD}$ scan was performed with a $10 \mu \mathrm{m}$ step size, with an exposure time of $600 \mathrm{~s}$ and a 
monochromator energy of $7.5 \mathrm{keV}(\lambda=0.165315 \mathrm{~nm})$. A fair agreement is found between the film delamination topology observed by optical microscopy (Fig. 3 (b)) and the measured X-ray stress maps. Figure 2 : (a) Optical microscope image of wrinkled regions close to a step in a $300 \mathrm{~nm}$ thin W film

(a)
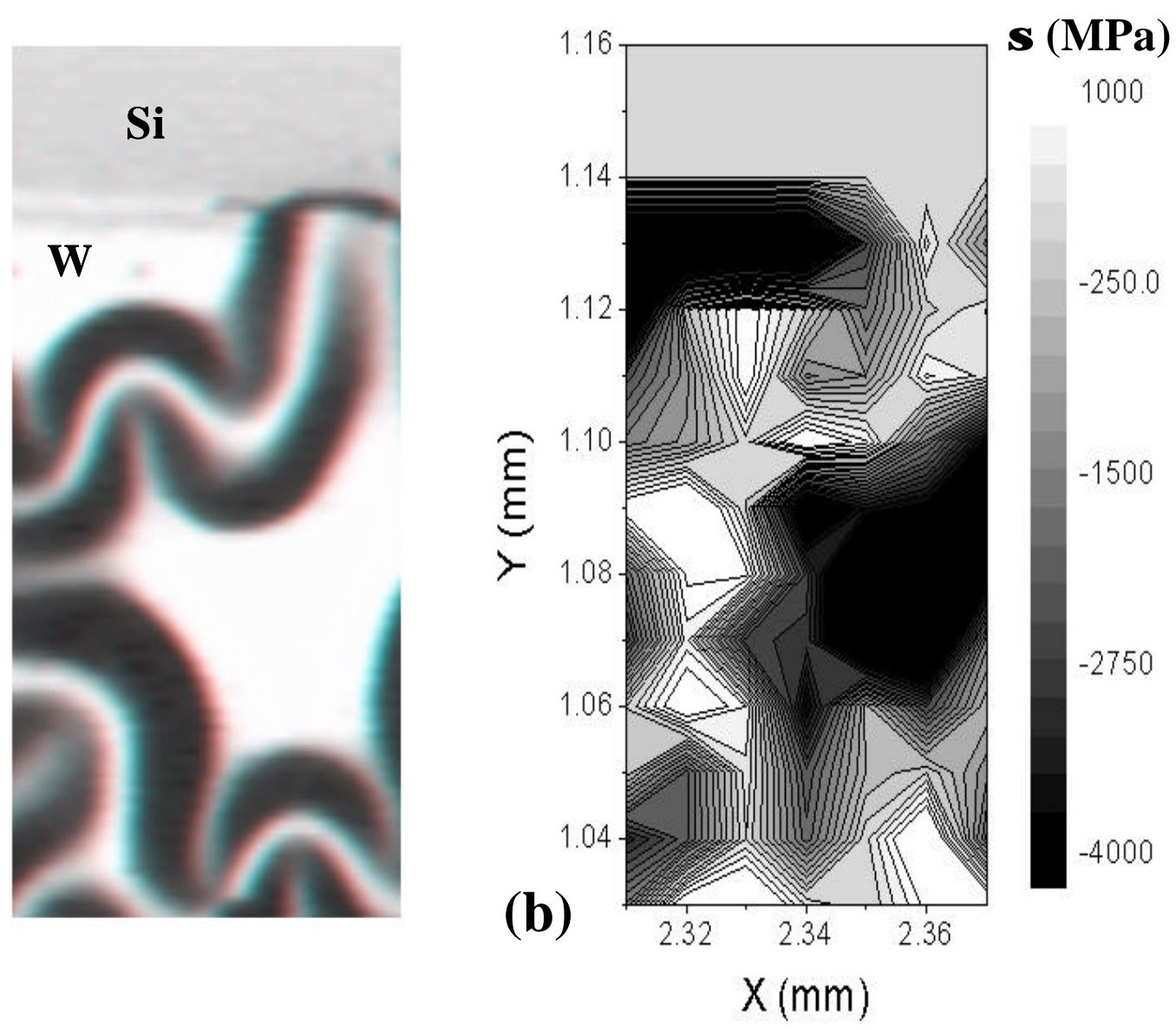

deposited on Si. (b) Corresponding stress map obtained by $\mu$ SXRD.

\section{Concluding remarks}

In conclusion, intragranular elastic properties of nanocrystalline tungsten thin films have been studied using an x-ray diffraction method combined with tensile testing. Microstructure and size effects on tungsten ingrain elastic constants have been evidenced. The variations of elastic constants can be correlated with the microstructure, in particular the stress-free lattice parameter. Further investigations will aim to find out interpretations of these experimental results in terms of interatomic potentials and cohesion energies.

Stress mapping measurements on thin film buckling at a micrometer scale have been successfully achieved [16]. A nearly stress-free state is measured at the top of the buckling in two different nanocrystalline materials and blister geometry whereas adherent regions are strongly compressed. Topology of film delamination areas observed by optical microscopy and the measured X-ray stress maps are similar. More precise x-ray measurements are engaged as well as Finite Element calculations of the stress field associated with 2D blisters and wrinkles in these metallic thin films. Confrontation of the stress profiles will permit to precise boundary conditions of the model and thus will lead to a better understanding of relevant parameters controlling thin film buckling. 


\section{Acknowledgements}

We would like to thank the beam line scientists M. Gailhanou, D. Thiaudière and E. Elkaïm for helpful assistance during synchrotron radiation experiments at LURE (Orsay, France) and Ph. Guerin for sample preparation. This work has received the financial support of the French Région Poitou-Charentes through 2000 annual scientific programs.

The Advanced Light Source is supported by the Director, Office of Science, Office of Basic Energy Sciences, Materials Sciences Division, of the U.S. Department of Energy under Contract No. DE-AC0376SF00098 at Lawrence Berkeley National Laboratory. We thank Intel Corp. for the partial funding of the end station.

\section{References}

[1] E.S. Machlin: Materials Science in Microelectronics: the relationships between thin film processing and structure (Gyro Press, USA 1995).

[2] V. Hauk: Structural and residual stress analysis by non destructive methods: evaluation, application, assessment (Elsevier, Netherlands 1997).

[3] J.L. Bassani, V. Vitek, and I. Alber: Acta metal. Mater. Vol. 40 (1992), p. S307

[4] J. Schiøtz, T. Vegge, F. D. Di Tolla and K. W. Jacobsen: Phys. Rev. B Vol. 60 (1999), p. 11971

[5] R. Danner, R.P. Huebener, C.S.L. Chun, M. Grimsditch and I.K. Schuller: Phys. Rev. B Vol. 33, (1986), p. 3696

[6] A. Fartash, E.E. Fullerton, I.K. Schuller, S.E. Bobbin, J.W. Wagner, R.C. Cammarata, S. Kumar and M. Grimsditch: Phys. Rev. B Vol. 44 (1991), p. 13760

[7] J. Pacaud, F. Martin, A. Michel, C. Jaouen, P. Djemia, and F. Ganot: Mat. Res. Soc. Symp. Proc. Vol. 615 (2001), p. G8.7

[8] P.-O. Renault, K.F. Badawi, L. Bimbault, and Ph. Goudeau, E. Elkaïm and J.P. Lauriat: Appl. Phys. Lett. Vol. 73 (1998), p. 1952

[9] K.F. Badawi, P. Villain, Ph. Goudeau and P.-O. Renault : Appl. Phys. Lett. Vol. 80 (2002), p. 4705

[10] P. Villain, Ph. Goudeau, P.-O. Renault and K.F. Badawi: Appl. Phys. Lett. Vol. 81 (2002), p. 4365

[11] G. Gioia and M. Ortiz: Advances in Applied Mechanics Vol. 33 (1997) p. 119

[12] A.A. MacDowell, R.S. Celestre, N. Tamura, R. Spolenak, B.C. Valek, W.L. Brown, J.C. Bravman, H.A. Padmore, B.W. Batterman and J.R. Patel: Nucl. Instrum. Methods Phys. Res. A Vol. 467-468 (2001), p. 936

[13] N. Tamura, R.S. Celestre, A.A. MacDowell, H.A. Padmore, R. Spolenak, B.C. Valek, N. Meier Chang, A. Manceau and J.R. Patel: Rev. of Scientific Instrum. Vol. 73 (2002), p. 1369

[14] N. Tamura, A.A. MacDowell, R.S. Celestre, H.A. Padmore, B.C. Valek, J.C. Bravman, R. Spolenak, W.L. Brown, T. Marieb, H. Fujimoto, B.W. Batterman and J.R. Patel: Appl. Phys. Lett. Vol. 80 (2002), p. 3724

[15] Ph. Goudeau, P. Villain, P.-O. Renault, N. Tamura, R.S. Celestre and H. Padmore: Materials Science Forum Vol. 404-407 (2002), p. 709

[16] Ph. Goudeau, P. Villain, N. Tamura and H. A. Padmore : Appl. Phys. Lett. (2003), submitted 